\title{
Gravitational radiation and the evolution of gravitational collapse in cylindrical symmetry
}

\author{
Alfonso García-Parrado ${ }^{\sharp *}$ and Filipe C. Mena ${ }^{b \natural \dagger}$ \\ \# Faculty of Mathematics and Physics, Charles University in Prague, V Holešovičkách 2, 18000 Praha 8, Czech Republic. \\ ${ }^{b}$ Centro de Matemática, Universidade do Minho, 4710-057 Braga, Portugal \\ ${ }^{\natural}$ Dep. Matemática, Instituto Superior Técnico, Universidade de Lisboa, Av. Rovisco Pais 1, 1049-001 Lisboa, Portugal
}

November 27, 2018

\begin{abstract}
Using the Sparling form and a geometric construction adapted to spacetimes with a 2-dimensional isometry group, we analyse a quasi-local measure of gravitational energy. We then study the gravitational radiation through spacetime junctions in cylindrically symmetric models of gravitational collapse to singularities. The models result from the matching of collapsing dust fluids interiors with gravitational wave exteriors, given by the Einstein-Rosen type solutions. For a given choice of a frame adapted to the symmetry of the matching hypersurface, we are able to compute the total gravitational energy radiated during the collapse and state whether the gravitational radiation is incoming or outgoing, in each case. This also enables us to distinguish whether a gravitational collapse is being enhanced by the gravitational radiation.
\end{abstract}

Keywords: Quasi-local energy; Gravitational waves; Sparling form

\section{Introduction}

The theorem about the positivity of the global gravitational field at spatial infinity is a cornerstone of General Relativity (GR) and there are already different proofs available of this important result. In practical applications, it would be desirable to have a quasi-local notion of energy which could be applied to finite objects. A great deal of effort has been put towards this goal 19, after the earlier proposals for the quasi-local mass by Hawking [12] and Penrose [16]. An interesting proposal was made by Brown and York 3] using a Hamiltonian formulation of General Relativity, and their definition depends on a gauge choice along three-dimensional spacelike hypersurfaces. Other authors have put forward definitions of mass which are based on more geometric constructions (see [22, 23] and references therein).

Associated to this problem is the problem of finding an appropriate Hamiltonian in each setting. In fact, the Hamiltonian for every diffeormorphism invariant theory depends on boundary conditions and is, therefore, non-unique. In General Relativity, for example, both the Komar Hamiltonian and the ADM Hamiltonian have been widely used (see e.g. 4). The latter, in turn, is a particular case of the Von Freud super-potential [10] and a problem related to the search of superpotentials for GR is the question of the existence of the Lanzcos potential for the Weyl tensor (see e.g. [6]).

An alternative approach to the definition of quasi-local gravitational energy quantities is given by the use of gravitational pseudo-tensors. In [9], Frauendiener explains how to treat pseudo-tensors in the geometric framework of the principal bundle defined by the frame bundle of a 4-dimensional Lorentzian manifold. Using this approach, it is shown that the energy-momentum pseudo-tensors of Einstein and Landau-Lifschitz can be recovered from pull-backs to the spacetime manifold under appropriate sections of the so-called Sparling 3-form, which is defined in the bundle of linear frames. Szabados [18, generalizes these results and gives explicit formulae for gravitational pseudo-tensors in rigid basis or anholonomic frames. In particular, he shows that the pull-backs defined by coordinate sections of the contravariant

\footnotetext{
*E-mail: alfonso@utf.mff.cuni.cz

†E-mail: fmena@math.uminho.pt
} 
and dual forms of the Sparling's form, defined on the bundle of linear frames over the spacetime, are the Bergman and the Landau-Lifshitz pseudo-tensors, respectively.

In most of the applications we are aware of, the quasi-local quantities are evaluated for an isolated system at infinity, while in the present work we are interested in their local values at certain physically interesting hypersurfaces such as spacetime junctions. Even though quasi-local gravitational energy quantities are well-defined, they are frame dependent and, therefore, whenever they are used it is necessary to give a justification about the choice of a particular frame. In the case of an isolated system, it is assumed that it is asymptotically flat and this means that one can introduce a group of asymptotic symmetries which, roughly speaking, correspond to the symmetries of the Poincaré group defined in flat spacetime or to a generalization called the BMS group. These asymptotic symmetries induce a set of privileged frames in the spacetime, in the sense that they correspond asymptotically to the generators of the asymptotic symmetry group and one can then take a frame of that set to compute a quasi-local gravitational energy quantity. Within this approach, it is possible to recover the standard ADM and Bondi notions of momentum for an isolated system using definitions of quasi-local quantities based on pseudo-tensors [11. An approach based on the study of the asymptotic conditions as boundary conditions, defining when an isolated system is radiating, is also possible [21.

We would like to carry over the formalism described in the paragraph above to the case of a system composed by an (inhomogeneous) interior matched to a radiating exterior through a common hypersurface. The objective here is to give measures of the gravitational energy interchanged at the matching hypersurface, which plays now a similar role to the spacelike or null infinity of the case of an isolated system. In the case of matching hypersurfaces with enough symmetry, there is a natural geometrical way to define frames adapted to the symmetries of the hypersurface, and which will play the role of the frames corresponding to the asymptotic symmetries used to compute the quasi-local gravitational energy at infinity in an isolated system.

Here, to formulate this problem in a mathematically precise way, we use a frame bundle formalism and define equivalence classes between frames. We then apply our formalism to spacetimes admitting a $G_{2}$ isometry group and, in particular, calculate the radiated gravitational energy through the transitivity hypersurfaces of such isometry groups. A novel aspect of our work is the introduction of the notion of gravitational collapse enhanced by the gravitational radiation, i.e. by the gravitational energy-momentum flux, defined using the Sparling form. The meaning of this notion is that during the collapse, gravitational radiation is produced and the gravitational energy can either be radiated outside of the collapsing body or be radiated inwards, in which case it enhances the gravitational collapse. Although our presentation is restricted to 4 dimensional spacetimes, most of it can be easily generalised to $n$ dimensions and can be applied e.g. to the brane world scenario where 4-dimensional branes are embedded, as hypersurfaces, on a 5-dimensional bulk.

Motivated by the recent detection of gravitational waves by LIGO [1, an interesting application of our formalism would be to models of objects with a finite radius in astrophysics, emitting gravitational radiation into a vacuum exterior. Due to Birkhoff's theorem, it is not possible to have such vacuum exteriors in spherical symmetry. The next simplest symmetry assumption is cylindrical symmetry. So, we will consider models of gravitational collapse in cylindrical symmetry. Such models result from gluing an interior metric representing matter collapsing gravitationally to an exterior metric containing gravitational waves. An interesting aspect of these models is that the matter density is non-zero at the matching boundary, resulting in discontinuities in the Einstein field equations. Even though a realistic system undergoing gravitational collapse is not cylindrically symmetric, some non-trivial effects already present in the real system can be found in the cylindrically symmetric model. For example, the degrees of freedom of the (pure) gravitational energy in the exterior may mix with the gravitational energy sourced by the matter at the boundary. These non-trivial effects may be captured by local, gauge-dependent, measures of gravitational energy.

The plan of the paper is as follows: in section 2, we revise some properties of the Sparling 3-form and we compute its transformation under a change of frame. In section 3, we review how to measure the gravitational energy radiated through embedded hypersurfaces of codimension 1 using the Sparling form. In section 4, we provide a geometric set-up for the definition of a frame based on the existence of a submanifold representing the surface of a radiative body. In section 5, we apply our formalism to cylindrically symmetric collapsing dust spacetimes containing gravitational radiation in the exterior which corresponds to the Einstein-Rosen solution.

Some algebraic computations of this paper have been done with $x A c t$ [15. We use Latin indices $a, b, c, . .=1,2,3,4$, Greek indices $\alpha, \beta, . .=1,2,3$, capitals $A, B, . .=1,2$ and units such that $8 \pi G=c=1$. 


\section{Sparling 3-form and Sparling identity}

In this section and in the following section 3, we review some known notions and properties about the Sparling form and the sparling identity that are needed for our work. Even though most of the material is known, we present the equations in accordance to the notation that shall be followed in sections 4 and 5.

Let $\left(\mathcal{M}, g_{a b}\right)$ be a 4 -dimensional spacetime with signature $(-,+,+,+)$ and consider the bundle of frames $L(\mathcal{M})$ over $\mathcal{M}$. Let $B \equiv\left\{\overrightarrow{\boldsymbol{e}}_{1}, \overrightarrow{\boldsymbol{e}}_{2}, \overrightarrow{\boldsymbol{e}}_{3}, \overrightarrow{\boldsymbol{e}}_{4}\right\}$ be a chosen frame (smooth section of $L(\mathcal{M})$ ) and denote by $B^{*} \equiv\left\{\boldsymbol{\theta}^{1}, \boldsymbol{\theta}^{2}, \boldsymbol{\theta}^{3}, \boldsymbol{\theta}^{4}\right\}$ its dual co-basis. Define the super-potential

$$
L\left(B, \vec{e}_{a}\right) \equiv \frac{1}{2} \eta_{a b c d} \boldsymbol{\theta}^{d} \wedge \Gamma^{b c}(B),
$$

where $\Gamma(B)_{c}^{b}$ is the connection 1-form on the frame $B$ and $\eta_{a b c d}$ are the components of the volume element of the metric $g_{a b}$ in the frame $B$. Note the dependency of the super-potential on both the frame $B$ and the frame elements $\overrightarrow{\boldsymbol{e}}_{a}$. 1 Under the choice of the frame $B$, the super-potential is a 2 -form in $\Lambda^{2}(\mathcal{M})$ and it fulfills the Sparling identity [9, 18, 5

$$
d\left(L\left(B, \overrightarrow{\boldsymbol{e}}_{a}\right)\right)=E\left(\overrightarrow{\boldsymbol{e}}_{a}\right)+S\left(B, \overrightarrow{\boldsymbol{e}}_{a}\right) .
$$

where the quantity $E\left(\overrightarrow{\boldsymbol{e}}_{a}\right) \in \Lambda^{3}(\mathcal{M})$ is the so-called Einstein 3-form, which is given in terms of the curvature 2 -form $R_{a b}$ by

$$
E\left(\overrightarrow{\boldsymbol{e}}_{a}\right)=-\frac{1}{2} \eta_{a b c d} \boldsymbol{\theta}^{b} \wedge R^{c d}
$$

and it has the property

$$
\boldsymbol{G}\left(\overrightarrow{\boldsymbol{e}}_{a}\right) \equiv * E\left(\overrightarrow{\boldsymbol{e}}_{a}\right)=G_{a b} \boldsymbol{\theta}^{b} \in \Lambda^{1}(\mathcal{M}),
$$

with $*$ representing the Hodge dual and $G_{a b}$ the Einstein tensor.

As $E\left(\overrightarrow{\boldsymbol{e}}_{a}\right)$ and $* E\left(\overrightarrow{\boldsymbol{e}}_{a}\right)$ are tensorial differential forms, we do not write their dependency on $B$, such dependency being only kept for pseudo-tensorial forms. This is for instance the case of the 3 -form $S\left(B, \overrightarrow{\boldsymbol{e}}_{a}\right)$ that it is given by

$$
S\left(B, \overrightarrow{\boldsymbol{e}}_{a}\right) \equiv \frac{1}{2}\left(\eta_{b c d e} \boldsymbol{\theta}^{b} \wedge \Gamma(B)_{a}^{c}-\eta_{a b c e} \boldsymbol{\theta}^{b} \wedge \Gamma(B)_{d}^{c}\right) \wedge \Gamma(B)^{d e}
$$

We will follow the convention of [9, 18] and call this form the Sparling 3-form. For us, the important point about the Sparling identity is that it can be interpreted as a conservation law in the following way: By defining the 1-form

$$
J\left(B, \overrightarrow{\boldsymbol{e}}_{a}\right) \equiv * d L\left(B, \overrightarrow{\boldsymbol{e}}_{a}\right),
$$

and computing the co-differential of $J\left(B, \vec{e}_{a}\right)$, we get

$$
\delta J\left(B, \overrightarrow{\boldsymbol{e}}_{a}\right)=-* d * J\left(B, \overrightarrow{\boldsymbol{e}}_{a}\right)=-* d * * d L\left(B, \overrightarrow{\boldsymbol{e}}_{a}\right)=0
$$

From this equation, we deduce that $J\left(B, \overrightarrow{\boldsymbol{e}}_{a}\right)$ always yields a conserved current, independently of the choice of the frame $B$ or the frame vectors $\overrightarrow{\boldsymbol{e}}_{a}$. According to the above considerations, the current $J\left(B, \overrightarrow{\boldsymbol{e}}_{a}\right)$ can be split into two parts as

$$
J\left(B, \overrightarrow{\boldsymbol{e}}_{a}\right)=\boldsymbol{G}\left(\overrightarrow{\boldsymbol{e}}_{a}\right)+* S\left(B, \overrightarrow{\boldsymbol{e}}_{a}\right) .
$$

The first part is a tensorial 1-form and, via the Einstein equations, can be interpreted as the flux of energy momentum due to the matter. The second part is non-tensorial and can be regarded as the flux of gravitational energy-momentum with respect to the frame $B$. If $\overrightarrow{\boldsymbol{u}}$ is a frame element of $B$ representing an observer, then the 1-form

$$
\mathfrak{j}(B, \overrightarrow{\boldsymbol{u}}) \equiv * S(B, \overrightarrow{\boldsymbol{u}})
$$

\footnotetext{
${ }^{1}$ One can define super-potential as a tensor valued form in the bundle of frames $L(\mathcal{M})$ as is done in 9 , 18 . To avoid introducing the notation and language used in fibre bundle theory, we adopt the point of view that the frame $B$ is a section of $L(\mathcal{M})$ and we can use this section to pull-back the equations in $L(\mathcal{M})$ to $\mathcal{M}$. In this way, forms and tensors have their usual meaning of fields in the manifold $\mathcal{M}$, but the price payed for this simplification is the appearance of an explicit dependency on the frame $B$ in certain expressions (the pseudo-tensors).
} 
shall be called the gravitational energy-momentum flux 1 -form for the observer $\overrightarrow{\boldsymbol{u}}$ with respect to the frame $B$ or just the gravitational flux 1-form, if no confusion arises. It can be interpreted as the gravitational energy-momentum flux, which in this context is also called gravitational radiation, measured by the given observer in the frame $B$. In our context, we need to be able to decide if gravitational radiation is leaving or entering a radiating source. To that end, we put forward the following definition.

Definition 1. We shall take the scalar quantity

$$
\mathfrak{j}(B, \overrightarrow{\boldsymbol{u}})\left\langle\overrightarrow{\boldsymbol{e}}_{a}\right\rangle
$$

as the component of the gravitational radiation flux along the direction defined by the vector field $\left(\overrightarrow{\boldsymbol{e}}_{a}\right)$. If this scalar quantity is positive (resp. negative), then we say that the gravitational radiation is outgoing (resp. incoming) with respect to $\left(\overrightarrow{\boldsymbol{e}}_{a}\right)$.

The fact that $\mathfrak{j}(B, \overrightarrow{\boldsymbol{u}})$ is frame and observer dependent does not render it useless when computing the radiated gravitational energy in certain cases, as we illustrate through explicit examples in section 5. In fact, in [9], it was proved that the scalar quantity (10) has a correspondence with the components of the energy-momentum complex given by Einstein in 1916 [7. Furthermore, it has been shown that, under an appropriate choice of frame $B$, it is possible to use this complex to recover the well-known notions of ADM and Bondi momentum at infinity for an isolated system (see [11]). Thus, following a similar approach, we will compute, in section 5, the gravitational energy radiated through a matching hypersurface separating two regions (exterior and interior) under the assumption that the hypersurface has certain symmetry properties. We will present explicit examples of systems undergoing gravitational collapse and show how the gravitational flux 1-form enables us to give a criterion stating whether gravitational radiation is being emitted or absorbed during the collapse.

It is obvious that a similar reasoning as the above can be performed if we take the Einstein form instead of the Sparling form. In this fashion, one can define the matter current with respect to the observer $\overrightarrow{\boldsymbol{u}}$ in the standard way

$$
\mathfrak{J}(\overrightarrow{\boldsymbol{u}}) \equiv \boldsymbol{G}(\overrightarrow{\boldsymbol{u}}) \in \Lambda^{1}(\mathcal{M}) .
$$

This current is tensorial and, as it is well-known, it represents the matter energy-momentum flux. From previous considerations, it is clear that the sum of both currents is always conserved, a property that can be expressed by the condition

$$
\delta(\mathfrak{J}(\overrightarrow{\boldsymbol{u}})+\mathfrak{j}(B, \overrightarrow{\boldsymbol{u}}))=0 .
$$

Interestingly, even though $\mathfrak{j}(B, \overrightarrow{\boldsymbol{u}})$ is frame dependent, the conservation property 12 holds for any frame $B$. A particular case occurs when each of the currents are independently conserved. As we will show, this is the situation for all the examples of section 5 modelling gravitational collapse. The implication of this is that, during the gravitational collapse, no fraction of the collapsing matter is transformed into gravitational radiation.

\section{Frame equivalence and radiated gravitational energy through an embedded hypersurface}

Suppose now that we introduce another frame $B^{\prime}$ and its associate co-frame $B^{\prime *}$, related to $B$ and $B^{*}$ through the formulae

$$
\overrightarrow{\boldsymbol{e}}_{a}^{\prime}=\gamma\left(B, B^{\prime}\right)_{a}{ }^{b} \overrightarrow{\boldsymbol{e}}_{b}, \quad \boldsymbol{\theta}^{\prime a}=\gamma\left(B^{\prime}, B\right)_{b}{ }^{a} \boldsymbol{\theta}^{b}, \quad \gamma\left(B, B^{\prime}\right)_{a}{ }^{b} \gamma\left(B^{\prime}, B\right)_{b}{ }^{c}=\delta_{a}{ }^{c},
$$

where the matrices $\gamma\left(B, B^{\prime}\right), \gamma\left(B^{\prime}, B\right)$ encode the transition between the frames $B$ and $B^{\prime}$. We regard these matrices as having entries which are scalars on $\mathcal{M}$ (0-forms). Under these circumstances, it is well-known that the connection 1-form transforms as

$$
\Gamma\left(B^{\prime}\right)_{b}^{a}=\gamma\left(B^{\prime}, B\right)_{q}^{a} \Gamma(B)_{p}^{q} \gamma\left(B, B^{\prime}\right)_{b}{ }^{p}+\gamma\left(B^{\prime}, B\right)_{p}{ }^{a} d\left(\gamma\left(B, B^{\prime}\right)_{b}{ }^{p}\right)
$$

We can use this expression to find the transformation law for the Sparling 3-form. A straightforward but tedious computation reveals: 
Lemma 1. Under the change of frames $B$ to $B^{\prime}$, the Sparling 3-form transforms as:

$$
\begin{aligned}
& S\left(B^{\prime}, \overrightarrow{\boldsymbol{e}}_{s}^{\prime}\right)=-\frac{1}{2} \gamma\left(B, B^{\prime}\right)^{r a} \eta_{a b c d} d \gamma\left(B, B^{\prime}\right)_{s}{ }^{b} \wedge d \gamma\left(B, B^{\prime}\right)_{r}{ }^{c} \wedge \theta^{d}- \\
& \frac{1}{2} \gamma\left(B^{\prime}, B\right)_{c}{ }^{h} \gamma\left(B, B^{\prime}\right)_{s}{ }^{a} \gamma\left(B, B^{\prime}\right)^{r b} \eta_{a b d m} d \gamma\left(B, B^{\prime}\right)_{r}{ }^{c} \wedge d \gamma\left(B, B^{\prime}\right)_{h}{ }^{d} \wedge \theta^{m}- \\
& \frac{1}{2} \eta^{a}{ }_{b c d} \Gamma(B)^{b}{ }_{a} \wedge d \gamma\left(B, B^{\prime}\right)_{s}{ }^{c} \wedge \theta^{d}-\frac{1}{2} \gamma\left(B^{\prime}, B\right)_{b}{ }^{r} \gamma\left(B, B^{\prime}\right)_{s}{ }^{a} \eta_{a}{ }^{c}{ }_{d m} \Gamma(B)^{b}{ }_{c} \wedge d \gamma\left(B, B^{\prime}\right)_{r}{ }^{d} \wedge \theta^{m}- \\
& \frac{1}{2} \gamma\left(B, B^{\prime}\right)_{s}{ }^{a} \gamma\left(B, B^{\prime}\right)^{r b} \eta_{b c d m} \Gamma(B)^{c}{ }_{a} \wedge d \gamma\left(B, B^{\prime}\right)_{r}{ }^{d} \wedge \theta^{m}+ \\
& \frac{1}{2} \gamma\left(B, B^{\prime}\right)_{s}{ }^{a} \gamma\left(B, B^{\prime}\right)^{r b} \eta_{a b c d} \Gamma(B)^{c}{ }_{m} \wedge d \gamma\left(B, B^{\prime}\right)_{r}{ }^{m} \wedge \theta^{d}+\gamma\left(B, B^{\prime}\right)_{s}{ }^{a} S\left(B, \overrightarrow{\boldsymbol{e}}_{a}\right) .
\end{aligned}
$$

From the expression above, it is clear that the relation between $S\left(B^{\prime}, \overrightarrow{\boldsymbol{e}}_{s}^{\prime}\right)$ and $S\left(B, \overrightarrow{\boldsymbol{e}}_{a}\right)$ is linear if $d \gamma\left(B, B^{\prime}\right)_{s}{ }^{a}=0$ and, in that case, $S$ transforms like a tensor. We shall explore this fact next.

Let $\mathcal{H} \subset \mathcal{M}$ be a co-dimension 1 smooth embedded hypersurface and consider two frames $B, B^{\prime}$ fulfilling the condition

$$
d\left(\phi^{*}\left(\gamma_{a}^{b}\left(B^{\prime}, B\right)\right)\right)=0,
$$

where $\phi: \mathcal{H} \rightarrow \mathcal{M}$ is a smooth embedding. Since one has that

$$
\phi^{*}\left(\gamma_{a}^{b}\left(B^{\prime}, B\right)\right)=\left.\gamma_{a}^{b}\left(B^{\prime}, B\right)\right|_{\mathcal{H}}
$$

we deduce that the scalars $\left.\gamma_{a}^{b}\left(B^{\prime}, B\right)\right|_{\mathcal{H}}$ can only be constants.

Proposition 1. For $\mathcal{H} \subset \mathcal{M}$ and $\phi: \mathcal{H} \rightarrow \mathcal{M}$ as described in the previous paragraph, the relation

$$
B \sim B^{\prime} \Longleftrightarrow d\left(\phi^{*}\left(\gamma_{a}^{b}\left(B^{\prime}, B\right)\right)\right)=0
$$

is an equivalence relation in $\mathfrak{X}(L(\mathcal{M}))$ (三 the set of smooth sections in the frame bundle $L(\mathcal{M})$ ).

Proof. To ease the notation, we suppress indices in the matrix $\gamma_{a}^{b}\left(B^{\prime}, B\right)$ and write it simply as $\gamma\left(B^{\prime}, B\right)$. We show that the properties characterizing an equivalence relation are fulfilled:

- Reflexive: As $\gamma(B, B)=I$ trivially $B \sim B$.

- Symmetric: If $B \sim B^{\prime}$ then $d\left(\phi^{*} \gamma\left(B^{\prime}, B\right)\right)=0$. One has then

$$
0=d\left(\phi^{*}(I)\right)=d\left(\phi^{*}\left(\gamma\left(B^{\prime}, B\right) \phi^{*}\left(\gamma\left(B, B^{\prime}\right)\right)\right)=\phi^{*}\left(\gamma\left(B^{\prime}, B\right)\right) d\left(\phi^{*}\left(\gamma\left(B, B^{\prime}\right)\right) .\right.\right.
$$

Since $\phi^{*}\left(\gamma\left(B^{\prime}, B\right)\right)$ is invertible, the last equation entails $d\left(\phi^{*}\left(\gamma\left(B, B^{\prime}\right)\right)=0\right.$ and thus $B^{\prime} \sim B$.

- Transitive: if $B \sim B^{\prime}$ and $B^{\prime} \sim B^{\prime \prime}$ then

$$
d\left(\phi^{*} \gamma\left(B^{\prime \prime}, B\right)\right)=d\left(\phi^{*}\left(\gamma\left(B^{\prime \prime}, B^{\prime}\right)\right) \phi^{*}\left(\gamma\left(B^{\prime}, B\right)\right)\right)=0 .
$$

Hence $B \sim B^{\prime \prime}$.

Proposition 2. If there are two frames $B, B^{\prime}$ on $\mathcal{M}$ such that $\left.B\right|_{\mathcal{H}}=\left.B^{\prime}\right|_{\mathcal{H}}$, then $B \sim B^{\prime}$.

Proof. If $\left.B\right|_{\mathcal{H}}=\left.B^{\prime}\right|_{\mathcal{H}}$, then eq. (17) holds with $\left.\gamma_{a}{ }^{b}\left(B^{\prime}, B\right)\right|_{\mathcal{H}}=\delta_{a}{ }^{b}$ and therefore $B \sim B^{\prime}$.

Now, let $B, B^{\prime}$ be frames such that $B \sim B^{\prime}$. Then

$$
\phi^{*}\left(d\left(\gamma_{a}^{b}\left(B^{\prime}, B\right)\right)\right)=d\left(\phi^{*}\left(\gamma_{a}^{b}\left(B^{\prime}, B\right)\right)\right)=0 .
$$

Using this information in 15 , we deduce

$$
\int_{\mathcal{H}} S\left(B^{\prime}, \overrightarrow{\boldsymbol{e}}_{a}^{\prime}\right)=\left.\gamma\left(B^{\prime}, B\right)_{a}{ }^{b}\right|_{\mathcal{H}} \int_{\mathcal{H}} S\left(B, \overrightarrow{\boldsymbol{e}}_{b}\right),
$$

where we used the fact that $\left.\gamma\left(B^{\prime}, B\right)_{a}{ }^{b}\right|_{\mathcal{H}}$ are constants as $B \sim B^{\prime}$. Bearing in mind this property, let us make the following definition

Definition 2. Let $\mathcal{H} \subset \mathcal{M}$ be an embedded co-dimension 1 smooth hypersurface. For a given frame $B$ and frame element $\overrightarrow{\boldsymbol{e}}_{a} \in B$, the gravitational flux through $\mathcal{H}$, with respect to $B$ and $\overrightarrow{\boldsymbol{e}}_{a}$ is defined as

$$
\boldsymbol{P}\left(B, \mathcal{H}, \overrightarrow{\boldsymbol{e}}_{a}\right) \equiv \int_{\mathcal{H}} S\left(B, \vec{e}_{a}\right) .
$$


If $B$ and $B^{\prime}$ are frames such that $B \sim B^{\prime}$, then eq. 21] can be rendered in the form

$$
\boldsymbol{P}\left(B, \mathcal{H}, \overrightarrow{\boldsymbol{e}}_{a}\right)=\left.\gamma\left(B^{\prime}, B\right)_{a}{ }^{b}\right|_{\mathcal{H}} \boldsymbol{P}\left(B^{\prime}, \mathcal{H}, \overrightarrow{\boldsymbol{e}}_{b}^{\prime}\right), \quad \text { for } \quad B \sim B^{\prime} .
$$

This means that if we have a privileged frame $B$ in our problem, we can compute the gravitational flux according to 22 and consider the scalars $\left\{\boldsymbol{P}\left(B, \mathcal{H}, \overrightarrow{\boldsymbol{e}}_{a}\right)\right\}$ as the components of the gravitational 4-momentum of the hypersurface $\mathcal{H}$. We shall represent it as $\boldsymbol{P}(B, \mathcal{H})$.

An important particular case occurs in vacuum, when $S\left(B, \overrightarrow{\boldsymbol{e}}_{a}\right)=d L\left(B, \overrightarrow{\boldsymbol{e}}_{a}\right)$. In this case, using Stokes theorem, we can write $(22)$ as follows

$$
\boldsymbol{P}\left(B, \mathcal{H}, \overrightarrow{\boldsymbol{e}}_{a}\right) \equiv \int_{\mathcal{H}} d L\left(B, \overrightarrow{\boldsymbol{e}}_{a}\right)=\int_{\partial \mathcal{H}} L\left(B, \overrightarrow{\boldsymbol{e}}_{a}\right) .
$$

In an asymptotically flat isolated system we can choose $\mathcal{H}$ in such a way that one of the connected components of $\partial \mathcal{H}$ surrounds the source. Denoting by $\Sigma_{r}$ such a connected component, we can study the existence of the limit

$$
\lim _{r \rightarrow \infty} \int_{\Sigma_{r}} L\left(B, \vec{e}_{a}\right)
$$

for a hypersurface $\Sigma_{r}$ that approaches infinity. This analysis has been already performed in a number of situations (see [1]):

1. The surface $\Sigma_{r}$ approaches spatial infinity. In this case, if we choose an asymptotically Cartesian frame $B$, one can show that the limit 25 corresponds to the ADM 4-momentum of the isolated source.

2. The surface $\Sigma_{r}$ approaches null infinity. In this case, if we choose a frame $B$ as the normalized translation defined by the asymptotic BMS group, then the limit 25 corresponds to the Bondi 4-momentum of the isolated source.

In any case, we note that the choice of a frame $B$ with suitable properties at infinity, yields integral values of quasi-local quantities that have a valid physical meaning. In the next section, we shall follow this approach and show how the choice of a frame $B$ adapted to the symmetries of our physical system, at the matching hypersurface, enables us to analyse the problem of the gravitational radiation through the matching boundary.

The most important point to stress here is that the matching hypersurface, in our case, plays a role similar to null infinity or spacelike infinity in the case of an isolated system. As discused above, the choice of a frame $B$ adapted to the symmetries of null or spacelike infinity (the asymptotic symmetries) provides, respectively, the Bondi or ADM momentum. Therefore, a frame $B$ adapted to the symmetries of the matching hypersurface, should give us the quantities corresponding to the flux of gravitational energymomentum through the matching hypersurface. The detailed analysis of this assertion is presented in the next section.

\section{Gravitational flux through a hypersurface in a spacetime with a $G_{2}$ isometry group}

Assume that $\mathcal{M}$ admits a maximal isometry group $G_{2}$ generated by the Killing vectors $\overrightarrow{\boldsymbol{\xi}}_{1}, \overrightarrow{\boldsymbol{\xi}}_{2}$. Let $\mathcal{H} \subset \mathcal{M}$ be a co-dimension 1 hypersurface. Choose a frame of the form

$$
B=\left\{\overrightarrow{\boldsymbol{n}}, \overrightarrow{\boldsymbol{\xi}}_{1}, \overrightarrow{\boldsymbol{\xi}}_{2}, \overrightarrow{\boldsymbol{u}}\right\}
$$

where $\overrightarrow{\boldsymbol{n}}$ is a vector field on $\mathcal{M}$ such that $\left.\overrightarrow{\boldsymbol{n}}\right|_{\mathcal{H}}$ is unit and normal to the hypersurface and $\overrightarrow{\boldsymbol{u}}$ is an unit timelike vector field representing an observer. This frame is fully adapted to the geometry of the matching hypersurface $\mathcal{H}$ because its elements are either fixed at $\mathcal{H}$ (as happens for an observer $\overrightarrow{\boldsymbol{u}}$ and the unit normal $\overrightarrow{\boldsymbol{n}}$ ) or are symmetries of the matching hypersurfaces (as happens for the Killing vectors $\overrightarrow{\boldsymbol{\xi}}_{1}, \overrightarrow{\boldsymbol{\xi}}_{2}$ ). There is a residual freedom in the choice of the frame $B$, coming from the fact that the vector field $\overrightarrow{\boldsymbol{n}}$ is defined up to a sign on $\mathcal{H}$ and that

$$
\overrightarrow{\boldsymbol{\xi}}_{1}^{\prime}=\alpha^{11} \overrightarrow{\boldsymbol{\xi}}_{1}+\alpha^{12} \overrightarrow{\boldsymbol{\xi}}_{2}, \quad \overrightarrow{\boldsymbol{\xi}}_{2}^{\prime}=\alpha^{21} \overrightarrow{\boldsymbol{\xi}}_{1}+\alpha^{22} \overrightarrow{\boldsymbol{\xi}}_{2}, \quad \alpha^{11}, \alpha^{12}, \alpha^{21}, \alpha^{22} \in \mathbb{R},
$$

are Killing vectors too. However, it is easy to get: 
Proposition 3. Any other frame $B^{\prime}=\left\{\overrightarrow{\boldsymbol{n}}, \overrightarrow{\boldsymbol{\xi}}_{1}^{\prime}, \overrightarrow{\boldsymbol{\xi}}_{2}^{\prime}, \overrightarrow{\boldsymbol{u}}\right\}$ with $\overrightarrow{\boldsymbol{\xi}}_{1}^{\prime}, \overrightarrow{\boldsymbol{\xi}}_{2}^{\prime}$ related to $\overrightarrow{\boldsymbol{\xi}}_{1}, \overrightarrow{\boldsymbol{\xi}}_{2}$ by 27) fulfills the property $B^{\prime} \sim B$.

From the previous proposition we deduce that, in a spacetime admitting a $G_{2}$ isometry group, one can define unambiguously the gravitational 4-momentum of a hypersurface $\mathcal{H}$ as $\boldsymbol{P}(B, \mathcal{H})$, where $B$ is a frame of the form (26). Since, in this case, the only freedom left to construct the frame $B$ is given by the choice of the observer $\overrightarrow{\boldsymbol{u}}$, we shall use the shorthand notation

$$
\boldsymbol{P}(\overrightarrow{\boldsymbol{u}}, \mathcal{H}) \equiv \boldsymbol{P}(B, \mathcal{H})
$$

Next, we are going to compute $\boldsymbol{P}(\overrightarrow{\boldsymbol{u}}, \mathcal{H})$ in a number of practical examples.

\section{Gravitational flux in cylindrically symmetric spacetimes}

In this section, we consider the problem of computing the gravitational flux through hypersurfaces in cylindrically symmetric spacetimes. The hypersurfaces, here, result from the matching of an exterior vacuum spacetime containing gravitational waves and a dust fluid interior spacetime which is collapsing to form a singularity. To simplify the presentation, we consider exact solutions to the Einstein equations with diagonal metrics only.

We start by briefly recalling the matching conditions between two spacetimes $\left(\mathcal{M}^{ \pm}, g^{ \pm}\right)$across timelike hypersurfaces $\mathcal{H}^{ \pm}$(see more details in [14]). The matching between two spacetimes requires an identification of their boundaries, i.e. a pair of embeddings $\Phi^{ \pm}: \mathcal{H} \longrightarrow \mathcal{M}^{ \pm}$with $\Phi^{ \pm}(\mathcal{H})=\mathcal{H}^{ \pm}$, where $\mathcal{H}$ is an abstract copy of one of the boundaries. Let $\xi^{\alpha}$ be a coordinate system on $\mathcal{H}$, where $\alpha, \beta=1,2,3$ are indices on the hypersurface. Given a vector basis $\left\{\partial / \partial \xi^{\alpha}\right\}$ of the tangent space $T \mathcal{H}$, the push-forwards $d \Phi^{ \pm}$provide a correspondence between the vectors of this basis and sets of linearly independent vectors tangent to $\mathcal{H}^{ \pm}$, given in appropriate coordinates by $e_{\alpha}^{ \pm a}=\partial_{\xi^{\alpha}} \Phi^{ \pm a}$. Here, we follow the convention of previous sections that small Latin letters $a, b=1,2,3,4$ represent spacetime indices. There are also (up to orientation) vectors $n_{ \pm}^{a}$ normal to the boundaries. The first and second fundamental forms on $\mathcal{H}$ are given by $q^{ \pm}=\Phi^{ \pm \star}\left(g^{ \pm}\right)$and $K^{ \pm}=\Phi^{ \pm \star}\left(\nabla^{ \pm} \mathbf{n}^{ \pm}\right)$, where $\Phi^{ \pm \star}$ denotes the pull-back corresponding to the maps $\Phi^{ \pm}$. In components, we may write

$$
\left.q_{\alpha \beta}^{ \pm} \equiv e_{\alpha}^{ \pm a} e_{\beta}^{ \pm b} g_{a b}\right|_{\mathcal{H}^{ \pm}}, \quad K_{\alpha \beta}^{ \pm}=-n_{a}^{ \pm} e_{\alpha}^{ \pm b} \nabla_{b}^{ \pm} e_{\beta}^{ \pm a} .
$$

The matching conditions between two spacetimes through $\mathcal{H}$ require the equality of the first and second fundamental form on $\mathcal{H}$, i.e.

$$
q_{\alpha \beta}^{+}=q_{\alpha \beta}^{-}, \quad K_{\alpha \beta}^{+}=K_{\alpha \beta}^{-} .
$$

In the examples below, the spacetimes are matched across cylinders of symmetry.

\subsection{Gravitational wave exteriors}

The most general diagonal metric for cylindrically symmetric vacuum spacetimes (with an Abelian $G_{2}$ acting on 2-dimensional spacelike hypersurfaces $S_{2}$ ) can be written as 13 .

$$
g^{+}=e^{2(\gamma-\psi)}(-d T \otimes d T+d \rho \otimes d \rho)+R^{2} e^{-2 \psi} d \tilde{\varphi} \otimes d \tilde{\varphi}+e^{2 \psi} d \tilde{z} \otimes d \tilde{z},
$$

where $\psi, \gamma, R$ are functions of the coordinates $\rho, T$ satisfying the Einstein equations 76 - 80 . This metric is written in coordinates adapted to the Killing vectors $\partial_{\tilde{\varphi}}$ and $\partial_{\tilde{z}}$. From the physical point of view, the metric models cylindrical gravitational waves with one polarization state and, for the choice $R(T, \rho)=\rho$, corresponds to the metric found by Einstein and Rosen [8]. So, we say that (31) are metrics of Einstein-Rosen type.

Dust matter sources to such spacetimes were studied in 20, 2, where the matching between collapsing interior spacetimes and Einstein-Rosen type of exteriors was shown to exist along timelike hypersurfaces. It would be therefore interesting to study the gravitational radiation through those hypersurfaces and to find out whether it enhances the gravitational collapse or not. It has been shown 20] that, in those cases, the matching hypersurfaces are ruled by geodesics and, from the point of view of the exterior, can be parametrized as

$$
\mathcal{H}^{+}:\left\{t=\lambda, \rho=r_{0}, \tilde{\varphi}=\phi, \tilde{z}=\zeta\right\},
$$

where $r_{0}$ is constant and $\lambda$ is a parameter along the geodesics. So, $\mathcal{H}^{+}$is generated by $\overrightarrow{\boldsymbol{e}}_{1}^{+}=\left.\partial_{t}\right|_{\mathcal{H}^{+}}, \overrightarrow{\boldsymbol{e}}_{2}^{+}=$ $\left.\partial_{\tilde{\varphi}}\right|_{\mathcal{H}^{+}}$and $\overrightarrow{\boldsymbol{e}}_{3}^{+}=\left.\partial_{\tilde{z}}\right|_{\mathcal{H}^{+}}$, which together with $\overrightarrow{\boldsymbol{n}}^{+}=\left.\partial_{r}\right|_{\mathcal{H}^{+}}$form an orthogonal basis for the spacetime at $\mathcal{H}^{+}$. 


\subsection{Spatially homogeneous interiors}

As interiors to (31), we first consider locally rotationally symmetric and spatially homogeneous dust solutions given by the Bianchi class $I$ of metrics [13], in cylindrical coordinates, as

$$
g^{-}=-d t \otimes d t+a(t)^{2} d z \otimes d z+b(t)^{2}\left(d r \otimes d r+r^{2} d \varphi \otimes d \varphi\right)
$$

with

$$
a(t)=(\alpha-t)^{-1 / 3}(\beta-t), \quad b(t)=(\alpha-t)^{2 / 3}
$$

for $0 \leq t<\min \{\alpha, \beta\}$ and $r \leq r_{0}$. If $\alpha=\beta$, the metric reduces to the Friedman-Lemaitre-RobertsonWalker (FLRW) class of spatially homogeneous and isotropic metrics.

The interior metrics (33) always contain cylinders of symmetry which are trapped near the singularity (see [20]). The marginally-trapped cylinders trace out a 3-surface in the interior which eventually arrives at the boundary of the matter located at $r=r_{0}$.

From the point of view of the interior, the matching hypersurface is parametrized as

$$
\mathcal{H}^{-}:\left\{T=\lambda, r=r_{0}, \varphi=\phi, z=\zeta\right\},
$$

so that $\mathcal{H}^{-}$is generated by $\overrightarrow{\boldsymbol{e}}_{1}^{-}=\left.\partial_{T}\right|_{\mathcal{H}^{-}}, \overrightarrow{\boldsymbol{e}}_{2}^{-}=\left.\partial_{\varphi}\right|_{\mathcal{H}^{-}}$and $\overrightarrow{\boldsymbol{e}}_{3}^{-}=\left.\partial_{z}\right|_{\mathcal{H}^{-}}$, which together with $\overrightarrow{\boldsymbol{n}}^{-}=\left.\partial_{\rho}\right|_{\mathcal{H}^{-}}$ form an orthogonal basis for the spacetime at $\mathcal{H}^{-}$.

Then, the matching conditions $(30)$ between $(31)$ and $(33)$ across $\mathcal{H}$ give

$$
e^{\psi} \stackrel{\underline{\mathcal{H}}}{=} a(t), \quad R \stackrel{\mathcal{H}}{=} a(t) b(t) r, \quad R_{\rho} \stackrel{\mathcal{H}}{=} a(t), \quad \gamma \stackrel{\mathcal{H}}{=} \psi, \quad \gamma_{\rho} \stackrel{\mathcal{H}}{=} \psi_{\rho}=0
$$

where $\stackrel{\mathcal{H}}{=}$ denotes evaluation at $\mathcal{H}$. In order to solve the matching problem, the strategy followed in [20] was as follows: For a suitable spacetime interior to (31) and for the conditions (35) at the boundary, one obtains an explicit solution for $R(\rho, T)$ which satisfies the wave equation (77) and the constraints 79 and $(80)$, at $\mathcal{H}$. In turn, the remaining Einstein equations 76 and $(78)$ can be seen as providing $\gamma, \rho \rho$ and $\psi_{, \rho \rho}$ on $\mathcal{H}$. Since we know data for the exterior metric and its normal derivatives at the boundary, it then follows [20] that a unique $\psi$ exists on a neighbourhood $\mathcal{D}$ of $\mathcal{H}$. Since $\gamma \stackrel{\mathcal{H}}{=} \psi$ and $\gamma_{, \rho} \stackrel{\mathcal{H}}{=} \psi, \rho$, once we have $\psi$, we use a similar argument in 76 to get a unique $\gamma$ in $\mathcal{D}$.

\subsubsection{FLRW case}

If the interior is given by a FLRW metric, then $a(t)=b(t)=(\alpha-t)^{2 / 3}$, for $t \in(-\infty, \alpha)$. From the exterior, the matching surface $\mathcal{H}$ is the cylinder $\rho=\rho_{0}, T<\alpha$, terminating in the singularity at $T=\alpha$. At $\mathcal{H}$, we have

$$
R \stackrel{\mathcal{H}}{=} r_{0}(\alpha-T)^{4 / 3}, \quad R_{\rho} \stackrel{\mathcal{H}}{=}(\alpha-T)^{\frac{2}{3}},
$$

so that, in particular, $R$ and $R_{\rho}$ on $\mathcal{H}$ are positive for $T<\alpha$, vanishing only at $T=\alpha$. We may obtain $R$ explicitly as

$$
R=\frac{r_{0}}{2}\left(\alpha+\rho_{0}-T-\rho\right)^{\frac{4}{3}}-\frac{3}{10}\left(\alpha+\rho_{0}-T-\rho\right)^{\frac{5}{3}}+\frac{r_{0}}{2}\left(\alpha-\rho_{0}-T+\rho\right)^{\frac{4}{3}}+\frac{3}{10}\left(\alpha-\rho_{0}-T+\rho\right)^{\frac{5}{3}}
$$

and we may also get

$$
\psi \stackrel{\mathcal{H}}{=} \frac{2}{3} \ln (\alpha-T), \quad \psi_{\rho} \stackrel{\mathcal{H}}{=} 0 .
$$

We now wish to calculate 28$]$ at $\mathcal{H}$ and, in order to do that, we need to choose an adequate frame in the exterior. Since $\psi=\gamma$ on $\mathcal{H}$, then the holonomic frame $B=\left\{\partial_{\tilde{\varphi}}, \partial_{\tilde{z}}, \partial_{\rho}, \partial_{T}\right\}$ is such that

$$
\left.\left\{\partial_{T}, \partial_{\rho}, \partial_{\tilde{z}}, \partial_{\tilde{\varphi}}\right\}\right|_{\mathcal{H}}=\left.\left\{e^{\gamma-\psi} \frac{\partial}{\partial T}, e^{\gamma-\psi} \frac{\partial}{\partial \rho}, \overrightarrow{\boldsymbol{\xi}}_{1}, \overrightarrow{\boldsymbol{\xi}}_{2}\right\}\right|_{\mathcal{H}},
$$

where the last frame has the properties of (26). Proposition 2 then implies that we can carry out our computations in the holonomic frame with the choice $\overrightarrow{\boldsymbol{u}}=\exp (\gamma-\psi) \frac{\partial}{\partial T}$ as the vector field representing the observer. In this case, a computation reveals that the Sparling 3-form at $\mathcal{H}$ is given by

$$
\left.S_{T}^{+}\right|_{\mathcal{H}}=-\frac{2 d T \wedge d \tilde{z} \wedge d \tilde{\phi}}{3(\alpha-T)^{\frac{1}{3}}},\left.\quad S_{\rho}^{+}\right|_{\mathcal{H}}=\frac{4 r_{0} d T \wedge d \tilde{z} \wedge d \tilde{\phi}}{9(\alpha-T)^{\frac{2}{3}}},\left.\quad S_{\tilde{z}}^{+}\right|_{\mathcal{H}}=0,\left.\quad S_{\tilde{\phi}}^{+}\right|_{\mathcal{H}}=0 .
$$


If we define now $\mathcal{H}^{+} \subset \mathcal{H}$ by the conditions $\tilde{z}_{0} \leq \tilde{z} \leq \tilde{z}_{0}+h, T_{0} \leq T \leq T_{1}$ with $\tilde{z}_{0}, T_{0}, T_{1}$ and $h$ constants, then one has

$$
\int_{\mathcal{H}^{+}} S_{T}^{+}=2 \pi h\left(\left(\alpha-T_{1}\right)^{\frac{2}{3}}-\left(\alpha-T_{0}\right)^{\frac{2}{3}}\right), \quad \int_{\mathcal{H}^{+}} S_{\rho}^{+}=\frac{8 \pi r_{0} h}{3}\left(\left(\alpha-T_{0}\right)^{\frac{1}{3}}-\left(\alpha-T_{1}\right)^{\frac{1}{3}}\right) .
$$

From this, we can compute the four real numbers which define the 4-momentum $\boldsymbol{P}\left(\overrightarrow{\boldsymbol{u}}, \mathcal{H}^{+}\right)$(see eq. $\left.22 \boldsymbol{\text { }}\right)$. Its components are

$$
\boldsymbol{P}\left(\overrightarrow{\boldsymbol{u}}, \mathcal{H}^{+}\right)=\left(2 \pi h\left(\left(\alpha-T_{1}\right)^{\frac{2}{3}}-\left(\alpha-T_{0}\right)^{\frac{2}{3}}\right), \frac{8 \pi r_{0} h}{3}\left(\left(\alpha-T_{0}\right)^{\frac{1}{3}}-\left(\alpha-T_{1}\right)^{\frac{1}{3}}\right), 0,0\right) .
$$

Also, we can compute the gravitational flux 1-form at the matching hypersurface according to definition 1 and check whether the gravitational radiation is incoming or outgoing with respect to the radial direction $\partial / \partial \rho$. The result is

$$
\mathfrak{j}(B, \overrightarrow{\boldsymbol{u}})\left\langle\frac{\partial}{\partial \rho}\right\rangle=\frac{2}{3 r_{0}(\alpha-T)^{\frac{5}{3}}} .
$$

It is interesting to note that the components of $\boldsymbol{P}\left(\overrightarrow{\boldsymbol{u}}, \mathcal{H}^{+}\right)$have a constant sign, namely, $P_{T}<0$ and

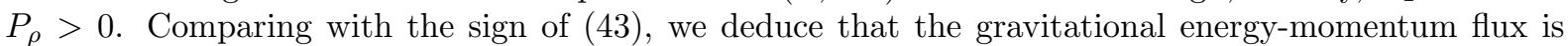
always outgoing. Even though one can also observe from $(40)$ that $\left.S_{T}^{+}\right|_{\mathcal{H}},\left.S_{\rho}^{+}\right|_{\mathcal{H}}$ diverge as $T \rightarrow \alpha$, the flux integrals remain finite. This last conclusion is in line with the conclusions of [20] using Weyl scalars.

As a consistency check, we can also carry out a similar computation for the interior, since the matching conditions entail $\mathcal{H}^{+}=\mathcal{H}^{-}=\mathcal{H}$ and $\boldsymbol{P}\left(\overrightarrow{\boldsymbol{u}}, \mathcal{H}^{+}\right)=\boldsymbol{P}\left(\overrightarrow{\boldsymbol{u}}, \mathcal{H}^{-}\right)$. To that end, we use the frame given by

$$
B=\left\{\partial_{t}, \frac{1}{b(t)} \partial_{r}, \partial_{z}, \partial_{\varphi}\right\},
$$

because this is the frame which matches at $\mathcal{H}$ with the frame used for the exterior, namely

$$
B=\left.\left\{\partial_{T}, \partial_{\rho}, \partial_{\tilde{z}}, \partial_{\tilde{\varphi}}\right\}\right|_{\mathcal{H}}=\left.\left\{\partial_{t}, \frac{1}{b(t)} \partial_{r}, \partial_{z}, \partial_{\varphi}\right\}\right|_{\mathcal{H}} .
$$

Under this frame choice, the Sparling 3-form in the interior is

$$
\begin{aligned}
S_{t}^{-} & =-\frac{2}{3(\alpha-t)^{1 / 3}} d t \wedge d z \wedge d \phi+\frac{4 r}{3(\alpha-t)^{2 / 3}} \boldsymbol{\theta}^{1} \wedge d z \wedge d \phi \\
S_{r}^{-} & =\frac{4 r}{9(\alpha-t)^{2 / 3}} d t \wedge d z \wedge d \phi-\frac{4}{3(\alpha-t)^{1 / 3}} \boldsymbol{\theta}^{1} \wedge d z \wedge d \phi, \\
S_{z}^{-} & =\frac{4 r}{3(\alpha-t)^{2 / 3}} d t \wedge \boldsymbol{\theta}^{1} \wedge d \phi, \quad S_{\phi}^{-}=\frac{4 r}{3(\alpha-t)^{2 / 3}} d t \wedge \boldsymbol{\theta}^{1} \wedge d z,
\end{aligned}
$$

where

$$
\boldsymbol{\theta}^{1}=(\alpha-t)^{2 / 3} d r
$$

The gravitational flux 1-form in the interior is then given by

$$
\mathfrak{j}\left(B, \frac{\partial}{\partial t}\right)={ }_{g^{-}}^{*}\left(S_{t}^{-}\right)=\frac{1}{3 r(\alpha-t)^{2}}(2(\alpha-t) d r-4 r d t),
$$

from which we get

$$
\mathfrak{j}\left(B, \frac{\partial}{\partial t}\right)\left\langle\frac{1}{(\alpha-t)^{\frac{2}{3}}} \frac{\partial}{\partial r}\right\rangle=\frac{2}{3 r(\alpha-t)^{\frac{5}{3}}},
$$

which agrees with $(43)$ at the matching hypersurface. If we assume now that $\mathcal{H}^{-} \subset \mathcal{H}$ is defined by the conditions $z_{0} \leq z \leq z_{0}+h, T_{0} \leq t \leq T_{1}$ with $z_{0}, T_{0}, T_{1}$ and $h$ constants, then one has

$$
\begin{aligned}
& \int_{\mathcal{H}^{-}} S_{t}^{-}=2 \pi h\left(\left(\alpha-T_{1}\right)^{\frac{2}{3}}-\left(\alpha-T_{0}\right)^{\frac{2}{3}}\right), \quad \int_{\mathcal{H}^{-}} S_{r}^{-}=\frac{8 \pi r_{0} h}{3}\left(\left(\alpha-T_{0}\right)^{\frac{1}{3}}-\left(\alpha-T_{1}\right)^{\frac{1}{3}}\right), \\
& \int_{\mathcal{H}^{-}} S_{z}^{-}=0, \quad \int_{\mathcal{H}^{-}} S_{\phi}^{-}=0 .
\end{aligned}
$$


Again, we compute the four real numbers which define the 4-momentum $\boldsymbol{P}\left(\overrightarrow{\boldsymbol{u}}, \mathcal{H}^{-}\right)$(see eq. 222$)$ ) The result is

$$
\boldsymbol{P}\left(\overrightarrow{\boldsymbol{u}}, \mathcal{H}^{-}\right)=\left(2 \pi h\left(\left(\alpha-T_{1}\right)^{\frac{2}{3}}-\left(\alpha-T_{0}\right)^{\frac{2}{3}}\right), \frac{8 \pi r_{0} h}{3}\left(\left(\alpha-T_{0}\right)^{\frac{1}{3}}-\left(\alpha-T_{1}\right)^{\frac{1}{3}}\right), 0,0\right) .
$$

Therefore, we can check explicitly that eqs. 42 and 51 are consistent with $\boldsymbol{P}\left(\overrightarrow{\boldsymbol{u}}, \mathcal{H}^{-}\right)=\boldsymbol{P}\left(\overrightarrow{\boldsymbol{u}}, \mathcal{H}^{+}\right)$.

Since the interior is non-vacuum, we can also compute the Einstein 3 -form with the result

$$
E_{t}=-\frac{4}{3} r d r \wedge d z \wedge d \phi, \quad E_{r}=E_{z}=E_{\phi}=0 .
$$

From (52), we easily deduce that $d E_{t}=0$ which implies that $d S_{t}=0$, so the matter energy-momentum current $\mathfrak{J}(\partial / \partial t)$ and $\mathfrak{j}(B, \partial / \partial t)$ are independently conserved currents.

\subsubsection{Bianchi $I$ case}

In this case, with equations (31)- $[35]$, we may find [20]

$$
\begin{aligned}
R= & \frac{r_{0}}{2}\left(\alpha+\rho_{0}-T-\rho\right)^{\frac{1}{3}}\left(\beta+\rho_{0}-T-\rho\right)-\frac{3}{4}\left(\alpha+\rho_{0}-T-\rho\right)^{\frac{2}{3}}\left(\beta+\rho_{0}-T-\rho\right) \\
& +\frac{9}{20}\left(\alpha+\rho_{0}-T-\rho\right)^{\frac{5}{3}} \\
& +\frac{r_{0}}{2}\left(\alpha-\rho_{0}-T+\rho\right)^{\frac{1}{3}}\left(\beta-\rho_{0}-T+\rho\right)+\frac{3}{4}\left(\alpha-\rho_{0}-T+\rho\right)^{\frac{2}{3}}\left(\beta-\rho_{0}-T+\rho\right) \\
& -\frac{9}{20}\left(\alpha-\rho_{0}-T+\rho\right)^{\frac{5}{3}},
\end{aligned}
$$

as well as

$$
\psi \stackrel{\mathcal{H}}{=} \gamma \stackrel{\mathcal{H}}{=} \ln (\beta-T)-\frac{1}{3} \ln (\alpha-T) .
$$

Using this information, we get

$$
\left.S_{T}^{+}\right|_{\mathcal{H}}=\frac{(\beta-T)-3(\alpha-T)}{3(\alpha-T)^{4 / 3}} d T \wedge d \tilde{z} \wedge d \tilde{\phi},\left.\quad S_{\rho}^{+}\right|_{\mathcal{H}}=2 r_{0} \frac{3(\alpha-T)-(\beta-T)}{9(\alpha-T)^{5 / 3}} d T \wedge d \tilde{z} \wedge d \tilde{\phi},
$$

the remaining components being zero. We define $\mathcal{H}^{+} \subset \mathcal{H}$ in a fashion similar as in subsection 5.2.1 and perform the following computations

$$
\begin{aligned}
\int_{\mathcal{H}^{+}} S_{T}^{+} & =2 \pi h\left[\frac{\beta-T_{1}}{\left(\alpha-T_{1}\right)^{\frac{1}{3}}}-\frac{\beta-T_{0}}{\left(\alpha-T_{0}\right)^{\frac{1}{3}}}\right], \\
\int_{\mathcal{H}^{+}} S_{\rho}^{+} & =\frac{2}{3} \pi h r_{0}\left[\frac{3\left(\alpha-T_{0}\right)+\left(\beta-T_{0}\right)}{\left(\alpha-T_{0}\right)^{2 / 3}}-\frac{3\left(\alpha-T_{1}\right)+\left(\beta-T_{1}\right)}{\left(\alpha-T_{1}\right)^{2 / 3}}\right] .
\end{aligned}
$$

Hence, we deduce that in this case:

$$
\boldsymbol{P}\left(\overrightarrow{\boldsymbol{u}}, \mathcal{H}^{+}\right)=2 \pi h\left(\frac{\beta-T_{1}}{\left(\alpha-T_{1}\right)^{\frac{1}{3}}}-\frac{\beta-T_{0}}{\left(\alpha-T_{0}\right)^{\frac{1}{3}}}, \frac{r_{0}}{3}\left[\frac{3\left(\alpha-T_{0}\right)+\left(\beta-T_{0}\right)}{\left(\alpha-T_{0}\right)^{2 / 3}}-\frac{3\left(\alpha-T_{1}\right)+\left(\beta-T_{1}\right)}{\left(\alpha-T_{1}\right)^{2 / 3}}\right], 0,0\right) .
$$

Using the gravitational flux 1-form at the matching hypersurface, we get

$$
\mathfrak{j}(B, \overrightarrow{\boldsymbol{u}})\left\langle\frac{\partial}{\partial \rho}\right\rangle={ }^{*}{ }_{g^{+}}\left(S_{T}^{+}\right)\left\langle\frac{\partial}{\partial \rho}\right\rangle=\frac{(\beta-T)-3(\alpha-T)}{3 r_{0}(\alpha-T)^{\frac{5}{3}}(T-\beta)} .
$$

One can carry out a similar computation from the interior, as we did in subsection 5.2.1, and use again the matching conditions to find that $\boldsymbol{P}\left(\overrightarrow{\boldsymbol{u}}, \mathcal{H}^{+}\right)=\boldsymbol{P}\left(\overrightarrow{\boldsymbol{u}}, \mathcal{H}^{-}\right)$. Also, the case $\alpha=\beta$ reduces to the FLRW case of the previous section.

Differently from the FLRW case, here one has to distinguish two cases: (i) $0<\alpha<\beta$, where the singularity is string-like along the axis of symmetry and $S_{T}, S_{\rho}$ diverge as $T \rightarrow \alpha$ and (ii) $0<\beta<\alpha$, where the spacetime singularity is pancake-like and $S_{T}, S_{\rho}$ are finite when $T \rightarrow \beta$. In the first case, the covector $\boldsymbol{P}\left(\overrightarrow{\boldsymbol{u}}, \mathcal{H}^{+}\right)$has components such that $P_{T}>0$ and $P_{\rho}<0$, whereas in the second case $P_{T}<0$ and 
$P_{\rho}<0$. Eq. (58) tells us that the gravitational flux with respect to the radial direction is incoming in the first case and outgoing in the second case. In the first case, $P_{T}$ is ever increasing as the gravitational collapse evolves towards the singularity and, therefore, $P_{T}$ represents a net gain of gravitational energy whereas, in the second case $P_{T}$, measures a net loss of gravitational energy. From this, we deduce that, in the first case, the gravitational collapse is enhanced by incoming gravitational radiation, whereas in the second case gravitational radiation is outgoing as the gravitational collapse progresses.

For the Bianchi $I$ interior, a computation shows that $\sqrt{52}$ is also valid. Therefore, $d E_{t}=0$, which implies that $d S_{t}=0$, so again the matter energy-momentum current $\mathfrak{J}(\partial / \partial t)$ and $\mathfrak{j}(B, \partial / \partial t)$ are independently conserved currents.

\subsection{Spatially inhomogeneous interior}

The following metric corresponds to an inhomogeneous solution of the Einstein equations of the Szekeres family with a dust source and a regular axis [17]:

$$
g^{-}=-d t \otimes d t+d r \otimes d r+\left(1-\frac{t^{2}+r^{2}}{\alpha^{2}}\right)^{2} d z \otimes d z+r^{2} d \varphi \otimes d \varphi,
$$

where $\alpha \in \mathbb{R} \backslash\{0\}$. As the previous two cases, one can also show that this metric is an interior to Einstein-Rosen type metrics [2] and it is, thus, an interesting case to study by comparison with the spatially homogeneous cases.

In this case, the equality of the first fundamental forms on $\mathcal{H}$ gives

$$
\gamma \stackrel{\mathcal{H}}{=} \psi, \quad R \stackrel{\mathcal{H}}{=} r_{0}\left(1-\frac{t^{2}+r_{0}^{2}}{\alpha^{2}}\right), \quad \psi \stackrel{\mathcal{H}}{=} \ln \left(1-\frac{t^{2}+r_{0}^{2}}{\alpha^{2}}\right), \quad \gamma_{, T} \stackrel{\mathcal{H}}{=} \psi_{, T}
$$

while the equality of the second fundamental forms gives

$$
\gamma_{, \rho} \stackrel{\mathcal{H}}{=} \psi_{, \rho}, \quad \psi_{, \rho} \stackrel{\mathcal{H}}{=}-\frac{2 r_{0}}{\alpha^{2}}\left(1-\frac{t^{2}+r_{0}^{2}}{\alpha^{2}}\right)^{-1}, \quad R_{, \rho} \stackrel{\mathcal{H}}{=} 1-\frac{t^{2}+3 r_{0}^{2}}{\alpha^{2}} .
$$

The solution to (77), which satisfies the matching conditions at $\mathcal{H}$, is

$$
\begin{aligned}
R(T, \rho) & =r_{0}\left(1-\frac{r_{0}^{2}}{\alpha^{2}}\right)+\left(1-\frac{3 r_{0}^{2}}{\alpha^{2}}\right)\left(\rho-\rho_{0}\right)-\frac{1}{6 \alpha^{2}}\left(\left(T+\rho-\rho_{0}\right)^{3}-\left(T-\rho+\rho_{0}\right)^{3}\right) \\
& -\frac{r_{0}}{2 \alpha^{2}}\left(\left(T-\rho+\rho_{0}\right)^{2}+\left(T+\rho-\rho_{0}\right)^{2}\right) .
\end{aligned}
$$

From the matching conditions $\psi \stackrel{\mathcal{H}}{=} \gamma$, we deduce that on $\mathcal{H}$

$$
\left.\left\{\partial_{\rho}, \partial_{\tilde{\varphi}}, \partial_{\tilde{z}}, \partial_{T}\right\}\right|_{\mathcal{H}}=\left.\left\{e^{\gamma-\psi} \frac{\partial}{\partial \rho}, \overrightarrow{\boldsymbol{\xi}}_{1}, \overrightarrow{\boldsymbol{\xi}}_{2}, e^{\gamma-\psi} \frac{\partial}{\partial T}\right\}\right|_{\mathcal{H}},
$$

where the last frame has the properties of (26). As we did in subsections 5.2.1 and 5.2.2, we appeal to Proposition 2 to carry out our computations in the holonomic frame $\left\{\partial_{T}, \partial_{\rho}, \partial_{\tilde{\varphi}}, \partial_{\tilde{z}}\right\}$, in order to compute (5) on $\mathcal{H}$. In this case, we actually carry out the computation in all of the exterior with the result:

$$
S_{T}^{+}=-\frac{2 T}{\alpha^{2}} d T \wedge d \tilde{z} \wedge d \tilde{\varphi}, \quad S_{\rho}^{+}=-\frac{2 r_{0}}{\alpha^{2}} d T \wedge d \tilde{z} \wedge d \tilde{\varphi}, \quad S_{\tilde{z}}^{+}=S_{\tilde{\varphi}}^{+}=0 .
$$

Next, we define $\mathcal{H}^{+} \subset \mathcal{H}$ and compute $\boldsymbol{P}\left(\overrightarrow{\boldsymbol{u}}, \mathcal{H}^{+}\right)$as we did before. The result is

$$
\boldsymbol{P}\left(\overrightarrow{\boldsymbol{u}}, \mathcal{H}^{+}\right)=\left(\frac{4 \pi h\left(T_{0}^{2}-T_{1}^{2}\right)}{\alpha^{2}}, \frac{4 \pi h r_{0}}{\alpha^{2}}\left(T_{0}-T_{1}\right), 0,0\right)
$$

At the matching hypersurface, we have

$$
\mathfrak{j}(B, \overrightarrow{\boldsymbol{u}})={ }^{*}{ }_{g^{+}}\left(S_{T}\right)=-\frac{2 T}{r_{0}\left(r_{0}^{2}-\alpha^{2}+T^{2}\right)} d \rho, \quad \mathfrak{j}(B, \overrightarrow{\boldsymbol{u}})\left\langle\frac{\partial}{\partial \rho}\right\rangle=-\frac{2 T}{r_{0}\left(r_{0}^{2}-\alpha^{2}+T^{2}\right)},
$$


from which we deduce that the gravitational flux is outgoing through the matching hypersurface. This indicates that the gravitational collapse is not enhanced by the gravitational radiation at the matching hypersurface.

In this particular case, we also supply an analysis of the collapsing process in the interior. To that end, we start by repeating the computation in the interior and find out whether the result agrees with what we have just found. In this case, the matching conditions entail

$$
\left.\left\{\partial_{T}, \partial_{\rho}, \partial_{\tilde{z}}, \partial_{\tilde{\varphi}}\right\}\right|_{\mathcal{H}}=\left.\left\{\partial_{t},-\partial_{r}, \partial_{z}, \partial_{\varphi}\right\}\right|_{\mathcal{H}}
$$

Comparing with $\sqrt[63]{6}$, we deduce that we can choose the frame $\left\{\partial_{t},-\partial_{r}, \partial_{z}, \partial_{\varphi}\right\}$ to carry out the computations in the interior. The result of these computations is

$$
S_{t}^{-}=-\frac{2 t}{\alpha^{2}} d t \wedge d z \wedge d \varphi, \quad S_{r}^{-}=-\frac{2 r_{0}}{\alpha^{2}} d t \wedge d z \wedge d \varphi, \quad S_{z}^{-}=S_{\varphi}^{-}=0 .
$$

So, this result yields

$$
\boldsymbol{P}\left(\overrightarrow{\boldsymbol{u}}, \mathcal{H}^{+}\right)=\boldsymbol{P}\left(\overrightarrow{\boldsymbol{u}}, \mathcal{H}^{-}\right),
$$

as expected.

The gravitational flux 1-form in the interior is explicitly given by

$$
\mathfrak{j}^{-}(B, \overrightarrow{\boldsymbol{u}})={ }^{*}{ }_{g^{-}}\left(S_{t}\right)=\frac{2(r d t+t d r)}{r\left(-\alpha^{2}+r^{2}+t^{2}\right)},
$$

To check whether the gravitational collapse is enhanced or not in the interior we choose to compute the gravitational radiation flux in the apparent horizon and, to that end, we analyse the trapped surface formation in the interior. In particular, we look at null 2-surfaces generated by the null vectors $\vec{k}^{( \pm)}=$ $\frac{\sqrt{2}}{2}\left(\partial_{t} \pm \partial_{r}\right)$. We then take the vectors $\vec{e}_{1}=\partial_{\phi}$ and $\vec{e}_{2}=\partial_{z}$, generators of the 2-cylinders and calculate the expansions $\theta_{A B}^{( \pm)}=-k_{a}^{( \pm)} e_{A}^{b} \nabla_{b} e_{B}^{a}$, where $A, B=1,2$, whose trace is denoted by $\theta^{( \pm)}$. The condition $\theta^{( \pm)}=0$, for the existence of a marginally trapped cylinder, is equivalent to

$$
\mathcal{T}: t^{2} \pm 2 t r+3 r^{2}=\alpha^{2},
$$

for $t^{2}+r^{2}<\alpha^{2}$. This means that, for a given $\alpha$, there exists a positive $t_{0}^{( \pm)}=\mp r+\sqrt{\alpha^{2}-2 r^{2}}$, for $r<\alpha / \sqrt{2}$, such that $\theta^{( \pm)}=0$ and cylinders to the future of $t_{0}^{(+)}$are trapped.

To carry out a more detailed analysis, we pick the branch with a positive sign and introduce a new variable $x$ through the definition $x \equiv t+r$. In terms of this new variable, condition (70) adopts the form $x^{2}+2 r^{2}=\alpha^{2}$, from which we conclude that in the $(x, r)$ plane, condition 70$)$ represents arcs of ellipses as shown in figure 1 . We can now eliminate the variable $t$ from (68). Next, using the map $x=\sqrt{\alpha^{2}-2 r^{2}}$, we compute the pullback of the Sparling 3-form to the marginally trapped cylinders which yields (here and in the following, we choose the positive sign in $(70)$ ):

$$
\begin{aligned}
& S_{t}^{-}=\frac{2\left(\alpha^{2}-4 r^{2}\right)}{\alpha^{2} \sqrt{\alpha^{2}-2 r^{2}}} d r \wedge d z \wedge d \varphi, \quad S_{r}^{-}=\frac{2\left(\alpha^{2}-2 r\left(\sqrt{\alpha^{2}-2 r^{2}}+2 r\right)\right)}{\alpha^{2} \sqrt{\alpha^{2}-2 r^{2}}} d r \wedge d z \wedge d \varphi, \\
& S_{z}^{-}=S_{\varphi}^{-}=0 .
\end{aligned}
$$

From these expressions, we can compute the gravitational flux 1-form on the marginally trapped cylinders using the standard definition, getting

$$
\mathfrak{j}^{-}(B, \overrightarrow{\boldsymbol{u}})=\frac{d r}{r^{2}\left(2 r^{2}-\alpha^{2}\right)}\left(\alpha^{2}-2 r\left(2 r+\sqrt{\alpha^{2}-2 r^{2}}\right)\right) .
$$

We can also compute the gravitational energy-momentum flux through these cylinders and we conclude that

$$
\int_{\mathcal{T}} S_{t}^{-}=\frac{4 \pi h r_{0} \sqrt{\alpha^{2}-2 r_{0}^{2}}}{\alpha^{2}}, \quad \int_{\mathcal{T}} S_{r}^{-}=\frac{4 \pi h r_{0}\left(\sqrt{\alpha^{2}-2 r_{0}^{2}}-r_{0}\right)}{\alpha^{2}} .
$$

Since $r_{0}<\alpha / \sqrt{2}$ we deduce, from the first integral, that the observer will measure a net positive emitted gravitational energy. However, looking at equation (73), we deduce that the gravitational flux with respect to $\partial / \partial r$ is incoming for $0<r<r_{*}$ and outgoing if $r_{*}<r<\alpha / \sqrt{3}$, where $r_{*}$ is given by

$$
r_{*} \equiv \frac{\alpha}{2} \sqrt{1-\frac{1}{\sqrt{3}}} .
$$



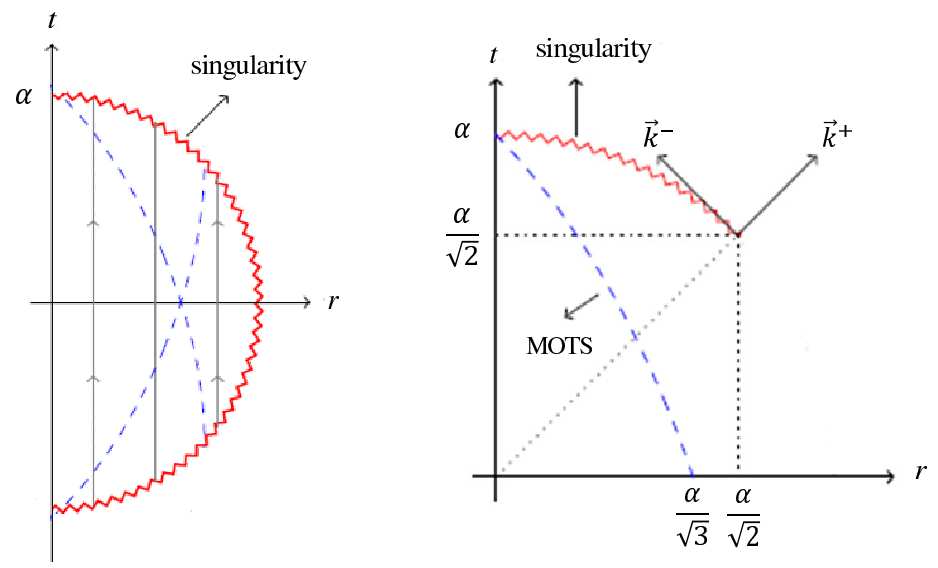

Figure 1: The left panel shows a diagram of the spacetime structure of (59), as obtained in [17]. The vertical lines represent the geodesics along which the dust moves between singularities. The blue dashed lines represent the marginally trapped cylinders which are arcs of elipses. On the right panel, a cut of the spacetime is depicted for $r<\alpha / \sqrt{2}$. This figure is adapted from [2].

Therefore, when the singularity is approached the radiation is incoming but when one gets towards the matching hypersurface the radiation is outgoing, consistently with the analysis carried out in the exterior. This means that the gravitational collapse will be enhanced by the gravitational radiation when the singularity is approached $\left(r<r_{*}\right)$ but not when $r>r_{*}$ (in particular, not in the matching hypersurface between the interior and the exterior).

To finish, we compute the Einstein 3-form in the interior obtaining

$$
E_{t}=-\frac{4}{\alpha^{2}} r d r \wedge d z \wedge d \phi, \quad E_{r}=E_{z}=E_{\phi}=0
$$

From this result, we deduce that $d E_{t}=0$ which entails $d S_{t}=0$, so in this case the matter energymomentum current $\mathfrak{J}^{-}(\partial / \partial t)$ and $\mathfrak{j}^{-}(B, \partial / \partial t)$ are also independently conserved currents in the interior.

\section{Conclusions}

We have considered a quasi-local measure of gravitational energy, using the Sparling 3-form and a geometric construction adapted to spacetimes with a 2-dimensional isometry group. We have then studied the gravitational energy-momentum flux in models of gravitational collapse with cylindrical symmetry.

Taking advantage of the existence of the Killing vectors, we defined a frame adapted to our problem to compute the gravitational radiation at the boundary and at the interior of the collapsing body. The interiors we have analysed contain a dust fluid and are FLRW, Bianchi $I$ and Szekeres solutions, whereas the exterior is always a vacuum Einstein-Rosen type solution containing gravitational waves. Our method shows that in the collapse modelled with the FLRW and inhomogeneous interiors, the gravitational radiation is always outgoing at the matching boundary of the collapsing body whereas, in the case of the Bianchi $I$, the gravitational radiation can be either outgoing or incoming at the boundary during the collapse.

We find that, in a model whose collapsing interior is a Bianchi $I$ spatially homogeneous spacetime with a string like singularity, the collapse is being enhanced by the gravitational radiation coming from the exterior. In the other cases analysed, the gravitational radiation is outgoing from the matching hypersurface during the collapse process. Note that these considerations pertain only to the analysis at the matching hypersurface. 
In the case of the inhomogeneous interior, we also carried out the analysis at the apparent horizon and found that, there, the gravitational collapse is enhanced towards its final phase, whereas it is not so at earlier stages. In this case, since the collapse is not enhanced at the matching hypersurface, we conclude that a gravitational energy-momentum flux is originated in the interior enhancing the collapse during its late evolution.

Our results show how a quasi-local measure of gravitational energy can be constructed in a geometrical way using the Sparling form, and can be applied to the problem of gravitational collapse of a fluid body having an exterior with gravitational waves.

\section{Acknowledgments}

We thank an anonymous referee for constructive criticisms on an earlier version of this paper. We thank FCT Projects Est-OE/MAT/UI0013/2014, PTDC/MAT-ANA/1275/2014 and CMAT, Univ. Minho, through FEDER Funds COMPETE. We also thank the Erwin Schrödinger International Institute for Mathematical Physics, ESI, where part of this work has been done. FCM thanks FCT for grant SFRH/BSAB/130242/2017. AGP thanks the financial support from Grant 14-37086G of the Czech Science Foundation and the partial support from the projects IT956-16 ("Eusko Jaurlaritza", Spain), FIS2014-57956-P ("Ministerio de Economía y Competitividad", Spain).

\section{Appendix}

The EFEs for the cylindrically symmetric vacuum metric (31) are

$$
\begin{aligned}
0 & =\gamma_{, T T}-\gamma_{, \rho \rho}-\psi_{, \rho}^{2}+\psi_{, T}^{2} \\
0 & =R_{, T T}-R_{, \rho \rho} \\
0 & =\psi_{, T T}+\frac{R_{, T}}{R} \psi_{, T}-\psi_{, \rho \rho}-\frac{R_{, \rho}}{R} \psi_{, \rho}
\end{aligned}
$$

together with the two constraint equations

$$
\begin{aligned}
\gamma_{, \rho} & =\frac{1}{R_{, \rho}^{2}-R_{, T}^{2}}\left(R R_{, \rho}\left(\psi_{, T}^{2}+\psi_{, \rho}^{2}\right)-2 R R_{, T} \psi_{, T} \psi_{, \rho}+R_{, \rho} R_{, \rho \rho}-R_{, T} R_{, T \rho}\right) \\
\gamma_{, T} & =-\frac{1}{R_{, T}^{2}-R_{, \rho}^{2}}\left(R R_{, T}\left(\psi_{, T}^{2}+\psi_{, \rho}^{2}\right)-2 R R_{, \rho} \psi_{, T} \psi_{, \rho}+R_{, T} R_{, \rho \rho}-R_{, \rho} R_{, T \rho}\right),
\end{aligned}
$$

where the commas denote differentiation.

\section{References}

[1] Abbott BP et al. (LIGO Scientific Collaboration and Virgo Collaboration), Observation of Gravitational Waves from a Binary Black Hole Merger, Phys. Rev. Lett. 116 (2016), 061102.

[2] I. Brito, M. F. A. Da Silva, F. C. Mena and N. O. Santos, Cylindrically symmetric inhomogeneous dust collapse with a zero expansion component, Class. Quant. Grav., 34 (2017), no. 20, 205005.

[3] J. D. Brown and J. W. York, Quasilocal energy and conserverd charges derived from the gravitational action, Phys. Rev. D, 47 (1993), no. 4, 1407-1419.

[4] P. T. Chruściel, On the relation between the Einstein and the Komar expressions for the energy of the gravitational field, Annales de l'I.H.P. Physique théorique, 42 (1985) 267-282.

[5] M. Dubois-Violette and J. Madore, Conservation laws and integrability conditions for gravitational and Yang-Mills field equations, Comm. Math. Phys., 108 (1987), no. 2, 213-223.

[6] S. B. Edgar and A. Höglund, The Lanczos potential for the Weyl curvature tensor: existence, wave equation and algorithms, Proc. R. Soc. A, 453 (1997) 835-851. 
[7] A. Einstein, Hamiltonsches Prinzip und Allgemeine Relativitätstheorie Sitzungsber. Preuss. Akad. Wiss. Phys. Math. Kl. 42 (1916) 1111-1116.

[8] A. Einstein and N. Rosen, On gravitational waves, Journal of the Franklin Institute, 223 (1937) $43-54$.

[9] J. Frauendiener, Geometric description of energy-momentum pseudotensors, Class. Quantum Grav., 6 (1989), no. 12, L237-L241.

[10] Von Ph. Freud, Über Die Ausdrücke Der Gesamtenergie Und Des Gesamtimpulses Eines Materiellen Systems in Der Allgemeinen Relativitatstheorie, Ann. Math., 40 (1939) 417-419.

[11] J. Goldberg, Invariant Transformations, Conservation Laws and Energy-Momentum, General Relativity and Gravitation, A. Held (ed.), Plenum, (1980), 469-488.

[12] S. W. Hawking, Gravitational Radiation in an Expanding Universe, J. Math. Phys., 9 (1968) 598-604.

[13] H. Stephani, D. Kramer, M. A. H. MacCallum, C. Hoenselaers and E. Herlt E, Exact Solutions of Einstein's Field Equations (Cambridge University Press, 2003).

[14] M. Mars and J. M. M. Senovilla, Geometry of general hypersurfaces in spacetime: junction conditions, Class. Quantum Grav., 10 (1993) 1865.

[15] J. M. Martín-García, xAct - efficient tensor computer algebra, http://www.xact.es.

[16] R. Penrose, Quasi-local mass and angular momentum in general relativity, Proc. R. Soc. A, 381 (1982) 53-63.

[17] J. M. M. Senovilla and R. Vera, Cylindrically symmetric dust spacetime, Class. Quantum Grav., 17 (2000) 2843-2846.

[18] L. B. Szabados, On canonical pseudotensors, Sparling's form and Noether currents, Class. Quantum Grav., 9 (1992), no. 12, 2521-2541.

[19] L. B. Szabados, Quasi-Local Energy-Momentum and Angular Momentum in GR: A Review Article, Liv. Rev. Relativity (2004) 7:4.

[20] P. Tod and F. C. Mena, Matching of spatially homogeneous non-stationary spacetimes to vacuum in cylindrical symmetry, Phys. Rev. D, 10 (2004) 104028.

[21] A. Trautman Radiation and Boundary Conditions in the Theory of Gravitation, Bulletin de l' Académie Polonaise des Sciences, Série des sci. math., astr. et phys. Vol. VI, No. 6 (1958) 407-412.

[22] M-T. Wang and S-T. Yau, Quasilocal mass in General Relativity, Phys. Rev. Lett., 102 (2009) 021101.

[23] M-T. Wang and S-T. Yau, Isometric Embeddings into the Minkowski Space and New Quasi-Local Mass, Comm. Math. Phys. 288 (2009) 919-942. 